\title{
REVIEW
}

\section{Arsenic trioxide as an anti-tumour agent: mechanisms of action and strategies of sensitization}

\author{
Yolanda Sánchez, Donna Amrán, Elena de Blas, Patricio Aller \\ Centro de Investigaciones Biológicas (CIB), Consejo Superior de Investigaciones Científicas (CSIC), Madrid, \\ Spain
}

Received $4^{\text {th }}$ March 2010.

Revised $11^{\text {th }}$ May 2010.

Published online $26^{\text {th }}$ May 2010.

\begin{abstract}
Summary
Arsenic trioxide $\left(\mathrm{As}_{2} \mathrm{O}_{3}, \mathrm{ATO}\right)$ is a very efficacious, clinically established agent for the treatment of acute promyelocytic leukaemia, and also potentially useful against other haematological and non-haematological malignancies. Nonetheless, the relative resistance of many tumour cell types requires the generation of sensitizing strategies. One of the properties of ATO which might be exploited for therapeutic purposes is its sensitivity to the intracellular oxidant state, as revealed by increased apoptosis production under conditions of reduced glutathione (GSH) depletion and/or elevated reactive oxygen species (ROS) content. This review summarizes some studies from our laboratory demonstrating that experimental modulation of protein kinase activities (PI3K/Akt, JNK, MEK/ERK) potentiates ATO-provoked apoptosis in relatively resistant human acute myeloid leukaemia (U937, HL60) cell lines by mechanisms involving GSH depletion and/or increased ROS content. In a similar manner, co-treatment with dietary flavonoides such as genistein, normally considered as anti-oxidants, may potentiate apoptosis via generation of moderate oxidative stress and activation of ROS-inducible protein kinases. Finally, co-treatment with ATO may sensitize otherwise refractory leukaemia cells to $\mathrm{TNF} \alpha$-family cytokine-produced apoptosis, by mechanisms involving the interplay between the "intrinsic" (mitochondrial) and "extrinsic" (death receptor-mediated) pathways.
\end{abstract}

Key words: arsenic trioxide; apoptosis; oxidative stress; protein kinases; phenolic agents; TNF $\alpha$; leukaemia cells

\section{INTRODUCTION}

Although the medical use of arsenicals is very ancient (for review see Miller et al. 2002), it was only recently that the anti-tumour importance of some these compounds was demonstrated beyond all doubt.

Patricio Aller, Centro de Investigaciones Biológicas, CSIC, Ramiro de Maeztu 9, 28040-Madrid, Spain

aller@cib.csic.es

둉 +34918373112 (ext. 4247)

圆 $\quad+34915360432$
Clinical assays carried out during the 1990s at Chinese (Harbin and Shanghai) medical institutions revealed that treatment with low, non-toxic doses of arsenic trioxide $\left(\mathrm{As}_{2} \mathrm{O}_{3}, \mathrm{ATO}\right)$ caused high rates of complete remission in patients suffering from acute promyelocytic leukaemia (APL), either newly diagnosed or relapsed after treatment with the more classical agent all-trans retinoic acid (ATRA) (reviewed by Wang and Chen 2008). Under the trademark of TRISENOX, ATO was later approved by the Food and Drug Administration in USA, and also recently by some European countries, as a highly efficacious and relatively safe agent to treat ATRA-refractory or relapsed APLs. Recent studies indicated that higher rates of durable remission may 
be still obtained using adequate ATO plus ATRA combinations (Wang and Chen 2008).

It is normally accepted that ATO efficacy is due to the capacity of the drug to disrupt the PML-RAR $\alpha$ fusion onco-protein, derived from the $\mathrm{t}(15 ; 17)$ chromosomal translocation, characteristic of most APLs. Since trivalent arsenicals easily react with vicinal sulfhydryl $(\mathrm{SH})$ residues in proteins, ATO probably binds a cysteine-rich region at the PML moiety, leading to PML-RAR $\alpha$ degradation. This would in turn relieve the APL cells from the differentiation blockade imposed by the fusion protein, driving them into terminal differentiation, the main response at low ATO concentrations (approx. $0.1-0.5 \mu \mathrm{M}$ in in vitro assays). At higher but still physiologically acceptable concentrations (approx. $0.5-2 \mu \mathrm{M})$ ATO preferentially induces apoptosis instead of differentiation. Nonetheless, it is doubtful that PML-RAR $\alpha$ disruption is the main cause for apoptosis generation. Actually, the drug exerts many other effects which, derived or not from ATO-cystein protein interactions, are potentially deleterious. As a few examples, ATO may cause reactive oxygen species (ROS) over-production, either from mitochondrial or extra-mitochondrial origin (respiratory chain inhibition, or activation of NADPH oxidase and NO synthase isozymes). ATO may also directly bind the adenine nucleotide translocator (ANT) at the mitochondrial permeability transition pore (mPTP) complex. Both ROS over-accumulation and direct binding may lead to MPTP opening, mitochondria dysfunction and apoptosis. In addition, ATO may elicit death receptor (DR5, TNF-R1) expression which facilitates apoptosis along the death-receptor mediated pathway (vide infra). ATO binds tubulin, disrupting the microtubule assembly and spindle formation, affecting cell cycle progression and viability. ATO may facilitate

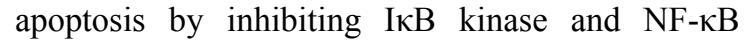
activation, by inhibiting JAK/STAT pathways, and by activating pro-apoptotic (JNK, p38) MAPK pathways. ATO may also disrupt other oncogenic fusion proteins, such as Bcr-Abl, characteristic of chronic myelogenous leukaemia, or AML1/MDS1/EVI1, present in some forms of leukaemia (for reviews see Miller et al. 2002, Wang and Chen 2008, Platanias 2009, among many others). Due to these and other effects, ATO causes apoptosis in cells from haematologic malignancies other than APL, such as myelodisplastic syndromes (MDS), multiple myeloma (MM), acute myeloid leukaemia (AML) and chronic myelogenous leukaemia (CML) (Amadori et al. 2005) and in solid tumour-derived cell lines. While the potential value of ATO is supported by clinical assays in some cases (e.g., MDS, MM), the applicability is restricted in other cases by the requirement of high toxic doses to effectively induce apoptosis. Thus, extending the therapeutic application of ATO would require the generation of sensitizing strategies, allowing decreasing the effective drug concentrations.

There are many excellent original studies and review articles dealing with historical, clinical, and mechanistic aspects of ATO, which are accessible to the reader but will not be commented on here. Instead, following editorial advice, the attention in this review will focus on some sensitizing approaches recently assayed in our laboratory.

\section{INDUCTION OF MODERATE OXIDATIVE STRESS AS A SENSITIZING STRATEGY}

A common characteristic of tumour cells is the presence of elevated ROS levels and oxidative damage to macromolecules, which are probably responsible for their oncogenic transformation and increased metabolic activity. On the other hand the intrinsic elevated level of oxidative stress makes tumour cells more susceptible than normal cells to the action of ROS-inducing cytotoxic agents, a property which may be exploited in anti-tumour therapies (Ozben 2007, Lau et al. 2008).

In addition to acting as a pro-oxidant agent itself, ATO is an oxidative stress- responsive drug - i.e., its toxicity depends very much on the oxidant state of the cells. For instance, ATO sensitivity in tumour cells inversely correlates with the inherent levels of GSH or GSH-associated enzymes (Dai et al. 1999, Yang et al. 1999). Due to the multiplicity of functions of glutathione, decreased GSH content may compromise essential defensive functions, such as ROS scavenging by glutathione peroxidases (leading to ROS over-accumulation) or ATO detoxification via glutathione S-transferases. Moreover, since GSH may directly bind and quench trivalent arsenic (Scott et al. 1993), a decrease in GSH may result in increased free intracellular ATO content and toxicity. In addition, the cell susceptibility to ATO toxicity directly correlates with the inherent intracellular ROS content (Yi et al. 2002). There are many studies demonstrating that experimental modulation of the oxidant environment, either by provoking GSH depletion (e.g. by treatment with the glutamate-cysteine ligase $(\gamma$-GCS $)$ inhibitor $\mathrm{D}$,L-buthionine-(S,R)-sulfoximine (BSO), or with ascorbic acid), or by co-treatment with exogenous $\mathrm{H}_{2} \mathrm{O}_{2}$ or ROS increasing agents, enhances ATO-provoked apoptosis in cultured cells and animal 
cell models (Dai et al. 1999, Pelicano et al. 2003, Maeda et al. 2004, Lau et al. 2008, and our unpublished observations) (see also Fig. 1). Interestingly, the potential efficacy of ATO/ascorbic acid combinations has been clinically assessed in phase II trials (Abou-Jawde et al. 2006).

The following paragraphs are dedicated to the description of two sensitizing strategies assayed in our laboratory in which oxidative stress seems to play a decisive regulatory role, namely modulation of protein kinase activities and co-treatment with dietary polyphenols. Experiments were mostly carried out in U937 and HL60 cells, two AML cell lines considered as relatively resistant to ATO toxicity.

\section{PROTEIN KINASE MODULATORS AS PRO-OXIDANT AND SENSITIZING AGENTS}

A frequent characteristic of tumour cells is the constitutive activation of defensive signalling pathways (e.g. PI3K/Akt, Raf/MEK/ERK, JAK/STAT), a property which may contribute to their abnormal elevated survival capacity and increased resistance to anticancer treatments. Hence, these pathways represent potential important targets for pharmacological intervention, and actually considerable efforts are being directed to the development of new compounds combining good inhibitory potency with physiological tolerance. There are many bibliographic examples demonstrating that experimental down-regulation of kinase activities increase the apoptotic efficacy of anti-tumour agents, including ATO (Tabellini et al. 2005, Ye et al. 2005, Altman et al. 2008, Lunghi et al. 2008, among others).

With these precedents in mind, we analyzed the capacity of some pharmacological PI3K/Akt and MAPK inhibitors to modulate the toxicity of ATO in U937 and other human acute myeloid leukaemia cell lines. In two successive articles (Ramos et al. 2005, 2006) we reported that co-treatment with non-toxic concentrations of PI3K/Akt(LY294002, wortmannin, AktiV), MEK/ERK (PD98059, U0126) and JNK (SP600125) inhibitors greatly increased ATO-provoked apoptosis, while the effects were poor or null when the inhibitors were combined with other anti-tumour drugs (e.g., the DNA-targeting agents cisplatin and camptothecin). Although not originally contemplated, we could also state that the kinase inhibitors decreased the intracellular GSH content (and when determined, the GSH/GSSG ratio) and increased intracellular ROS over-accumulation, which were at least in part responsible for apoptosis potentiation (see some results, Fig. 1). Mechanistic studies revealed that GSH depletion could be regulated at different levels. Thus, PI3K and JNK inhibitors down-regulated $\gamma$-GCS activity, in similar manner as the canonical GSH suppressor BSO. In contrast, MEK/ERK inhibitors caused $\gamma$-GCS-independent, N-acetyl-L-cysteine (NAC)-preventable GSH depletion, suggesting regulation at the level of substrate (cysteine) availability. It must be noted that most current bibliographic information indicates modulation (generally stimulation) of protein kinase activities by oxidative stress, although some reports suggest that the inverse cause-effect relationship, namely modulation of oxidative stress parameters by protein kinases, is also possible. For instance, it was described that $\gamma$-GCS activity is susceptible of modulation by changes in phosphorylation (Sun et al. 1996), and that PI3K inhibitors suppressed insulin-provoked increase in $\gamma$-GCS synthesis in rat hepatocytes (Kim et al. 2004), two observations that are fully compatible with our results. Of note, our findings revealing that the potentiation of ATO-provoked apoptosis by MEK/ERK and JNK inhibitors is mediated by GSH depletion have recently been corroborated by other authors (Lin et al. 2009).

Of course, multiple factors other than oxidative stress may participate in the potentiation of ATO-provoked apoptosis by protein kinase modulators. For instance, Lunghi et al. (2008) reported that MEK/ERK inhibitors potentiated ATO-provoked apoptosis in multiple myeloma cells deficient for p53 (a situation similar to HL60 and U937 AML cells) via increased expression of pro-apoptotic Bim and death receptors (DRs) 4 and 5. Nonetheless, some relationship of these findings with oxidative stress might be possible, since other authors also reported involvement of DR5 over-expression in ATO-provoked apoptosis under conditions of GSH depletion in U937 and other leukaemia cells (Chen et al. 2006). In addition, the relationship linking protein kinase down-regulation with generation of oxidative stress is not univocal, but may depend on the experimental conditions. For instance, Sordet et al. (2001) indicated that 12-O-tetradecanoylphorbol-13-acetate (TPA)-treated, differentiated U937 cells exhibited increased susceptibility to apoptosis induction by ATO, and that this effect was associated with a decrease in GSH content and an increase in ROS accumulation. In a later study (Fernández et al. 2004) we corroborated these results using two PKC activators, TPA and bryostatin 1, and also demonstrated that TPA- or bryostatin 1-provoked GSH decrease and apoptosis potentiation were preceded and mediated by ERK activation. Thus, 

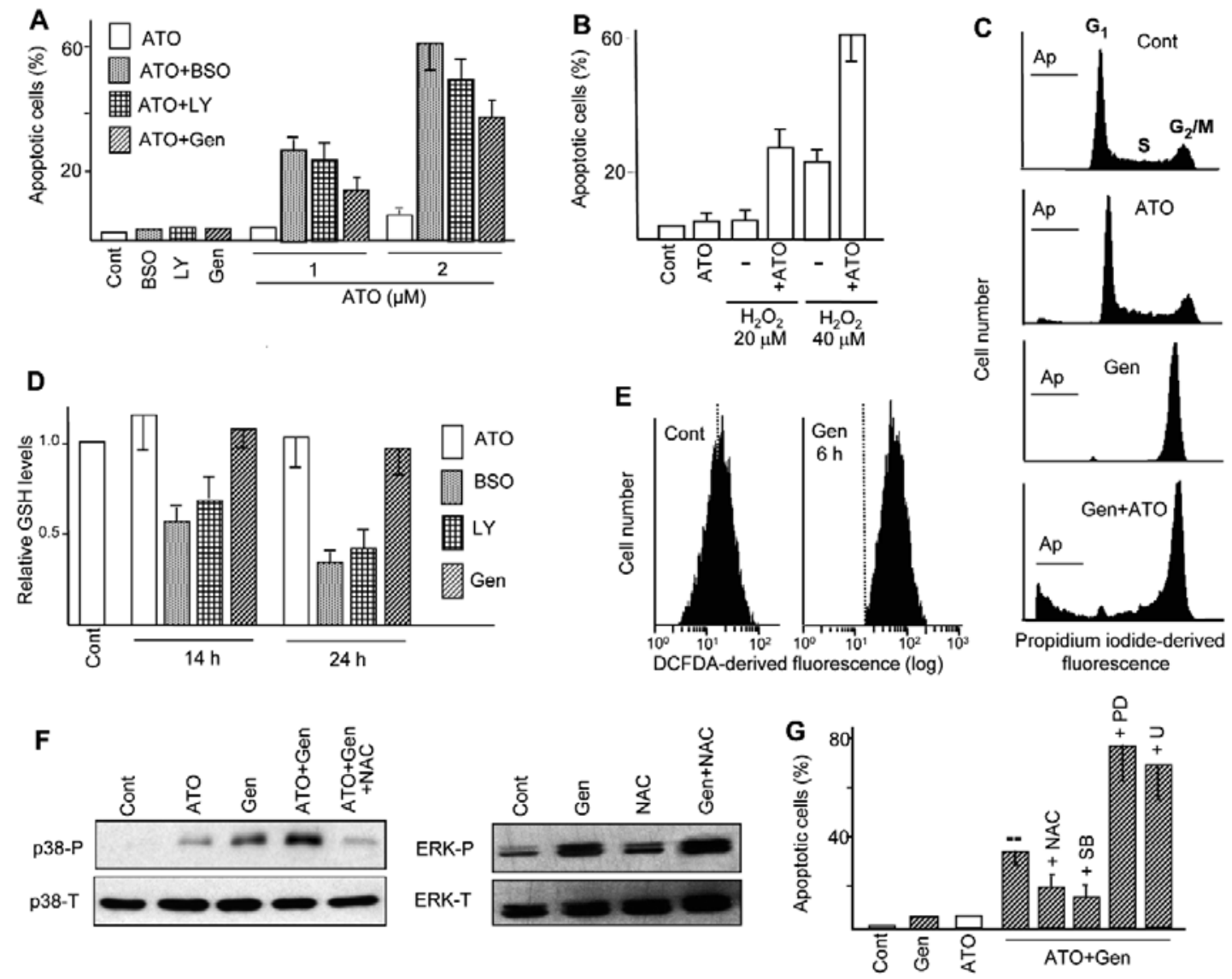

Fig. 1. Potentiation of ATO-provoked apoptosis by pro-oxidant agents. (A, B) Frequency of apoptosis, as determined by chromatin condensation/fragmentation, in U937 cell cultures treated for $24 \mathrm{~h}$ with the GSH suppressing agent DL-buthione-(S,R)-sulfoximine (BSO, $1 \mathrm{mM}$ ), the PI3K inhibitor LY294002 (LY, 30 $\mu \mathrm{M})$, the isoflavone genistein $(\mathrm{Gen}, 50 \mu \mathrm{M})$, low $\mathrm{H}_{2} \mathrm{O}_{2}$ concentrations, and ATO $(2 \mu \mathrm{M}$, except when otherwise indicated $)$, alone and in combination. Observe that the effects in combined treatments are higher than those caused by each agent alone. (C) Cell cycle distribution and frequency of apoptotic cells (Ap) at $24 \mathrm{~h}$ of treatment, as determined by propidium iodide staining and flow cytometry assays, in cultures treated with ATO and genistein, alone and in combination. (D) Relative intracellular GSH content, as measured by monochlorobimane derivatization and fluorimetry determination, 14 and $24 \mathrm{~h}$ of treatment with the indicated agents. The value 1 was arbitrarily given to untreated (Cont) cells. (E) Increase intracellular ROS content upon treatment with genistein, as determined by labelling with $\mathrm{H}_{2}$ DCFDA and fluorescence measurement by flow cytometry. (F) Relative levels of phosphorylated (P) and total (T) p38-MAPK and ERK, as determined at $16 \mathrm{~h}$ of treatment by immunoblot. Observe that genistein stimulates phosphorylation/activation of both kinases, but only p38-MAPK is dependent on oxidative stress, as indicated by the inhibitory action of the antioxidant agent $\mathrm{N}$-acetyl-L-cysteine (NAC, $10 \mathrm{mM}$ ). (G) Modulation of ATO plus genistein-provoked apoptosis by NAC, the p38-MAPK inhibitor SB203580 (SB, $10 \mu \mathrm{M})$, and the MEK/ERK inhibitors PD98059 (PD, $20 \mu \mathrm{M})$ and U0126 (U, $2.5 \mu \mathrm{M})$. Observe that NAC and SB203580 exert a protective action, indicating that apoptosis induction is mediated by oxidative stress and p38-MAPK. On the other hand PD98059 and U0126 over-increase apoptosis generation, indicating a protective action of ERKs.

while these results corroborate the importance of oxidative stress in cell sensitization to ATO-provoked apoptosis, they also indicate that oxidative stress is compatible with two opposite modes of ERK modulation, namely down-regulation by pharmacological kinase inhibitors and up-regulation by PKC activators.

\section{DIETARY POLYPHENOLS AS PRO-OXIDANT AND SENSITIZING AGENTS}

Flavonoids and other plant-derived phenolic agents represent a large group of compounds present in our daily diet. In the low concentrations obtainable from food, polyphenols normally exert beneficial 
chemo-preventive and anti-inflammatory effects, which are attributed to their anti-oxidant capacities (Rahman et al. 2006). Nonetheless at higher (but still physiologically tolerable) concentrations these agents may cause cytoreduction by inducing tumour cell apoptosis. The anti-tumour capacity of polyphenols was demonstrated under cell culture conditions, but also corroborated in vivo using mouse models (e.g., using genistein, Mohammad et al. 2006) and clinical assays in humans (e.g., curcumin, reviewed by Anand et al. 2008). Because of this, and also because they are better tolerated by the organism than most conventional anticancer drugs, the possibility of incorporating natural phenolic agents in clinical protocols has attracted great attention. Anyway, rather than as monotherapy, polyphenols may be more efficacious when used as radio- and chemo-sensitizing agents, in combination with conventional antitumour agents (Garg et al. 2005, Sarkar and Li 2006, Limtrakul 2007). The pro-apoptotic action of polyphenols is normally attributed to their capacity to down-regulate defensive, anti-apoptotic factors, such as NF- $\kappa \mathrm{B}$ and the PI3K/Akt pathway (Aggarwall and Shishodia 2006, Ramos 2008). Nonetheless, it must be noted that in spite of their normal anti-oxidant functions, some polyphenols may act as pro-oxidant agents, causing ROS over-production from either mitochondrial (Salvi et al. 2002) or extra-mitochondrial (Galati et al. 2002) sources. The physiological and perhaps therapeutical consequences of polyphenol-provoked oxidative stress have been insufficiently explored.

In a recent publication (Sánchez et al. 2008) we demonstrated that co-treatment with the soy-derived isoflavone genistein greatly increased ATO-provoked apoptosis in human myeloid and lymphoid leukaemia cell lines. This represented a somewhat drug- and tumour cell-selective response, since isoflavoneprovoked sensitization was poor or null in combination with other anti-tumour agents (e.g., the DNA-targeting drug cisplatin), or when combined with ATO in non-tumour proliferating peripheral blood lymphocytes. Time-course studies revealed that within the time-period required for apoptosis execution (16-24 h) genistein did not affect NF- $\mathrm{kB}$ or Akt activation, which were only down-regulated at later times ( $48 \mathrm{~h}$ and thereafter). On the other hand, the isoflavone caused a rapid (3-6 h) and moderate ROS over-accumulation which, as indicated by the protective action of anti-oxidant agents, was at least in part responsible for the pro-apoptotic response. Moreover, genistein caused ROS-dependent activation of p38-MAPK and AMPK kinases which, as indicated by the protective action of pharmacological inhibitors or specific siRNAs, also mediated apoptosis potentiation (some of these results are presented in Fig. 1). It is noteworthy that genistein also caused ROS-independent activation of the Raf-1/MEK/ERK pathway. However this pathway did not promote but instead restrained apoptosis, since co-treatment with pharmacological MEK/ERK inhibitors further increased the toxicity ATO plus genistein (Sánchez et al. 2009a). While ROS over-production also mediate the potentiation of ATO-provoked apoptosis by other polyphenols - e.g., epigallocatechin-3-gallate [EGCG], naringenin and curcumin (Nakazato et al. 2005, Sánchez et al. 2009b, and our unpublished results) - this is not the only possible mechanism responsible for ATO sensitization. For instance, co-treatment with the flavonol quercetin greatly potentiated ATO-provoked apoptosis in U937 leukaemia cells, in the absence of measurable ROS over-production, and without apparent protection by ROS-scavenging agents. Nevertheless, quercetin rapidly caused Akt dephosphorylation/inactivation (16 h) together with slight GSH depletion (Ramos and Aller 2008), a response which as demonstrated above suffices to increase ATO toxicity.

Although not directly within the scope of this review, it may be worthy of mention that phenolic agents cause multiple molecular and cellular effects other than apoptosis, but also with potential therapeutic benefits. For instance, genistein inhibits cell proliferation by causing $\mathrm{G}_{2} / \mathrm{M}$ cycle phase arrest. Indirect determinations suggested that genistein-provoked $\mathrm{G}_{2} / \mathrm{M}$ blockade is probably derived from its action as a DNA topoisomerase II inhibitor, while the pro-oxidant and pro-apoptotic responses of the isoflavone are apparently associated with its capacity to inhibit tyrosine kinases (Sánchez et al. 2009b, and references therein). In addition, genistein induces differentiation in human AML cell lines, a response which in contrast to the pro-apoptotic action of the isoflavone is not regulated by ROS and p38-MAPK, but is dependent on persistent Raf-1/MEK/ERK activation (Sánchez et al. 2009a). Importantly, this study also indicated cooperative effects between genistein and ATRA, namely potentiation of ATRA-provoked differentiation by low genistein concentrations, and prevention by ATRA of the long-term toxic effects of the isoflavone. Hence, the potential application of genistein and perhaps other related phenolic agents in differentiation therapies deserves to be considered. 
A
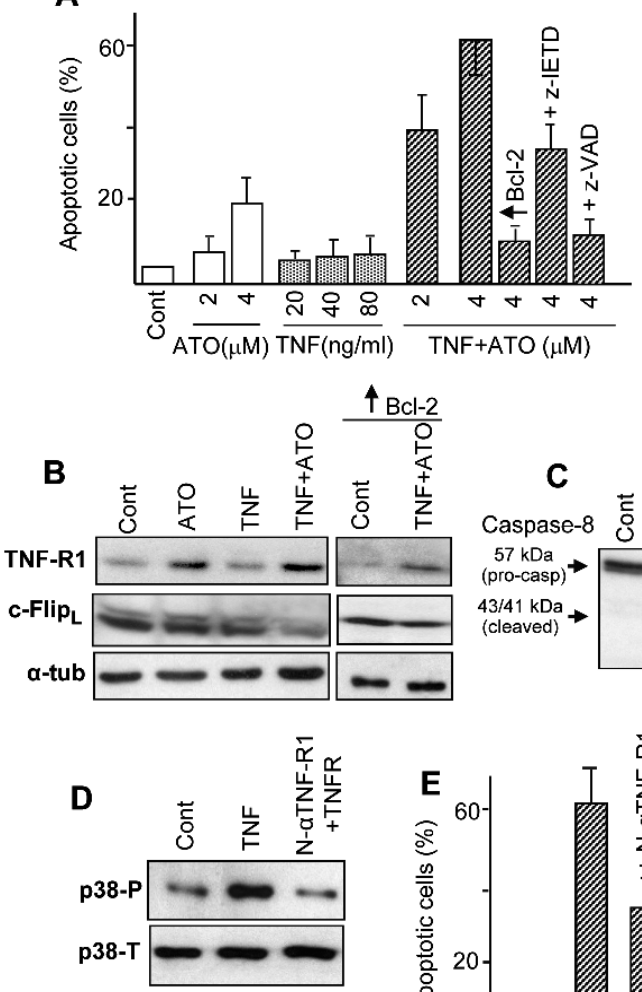

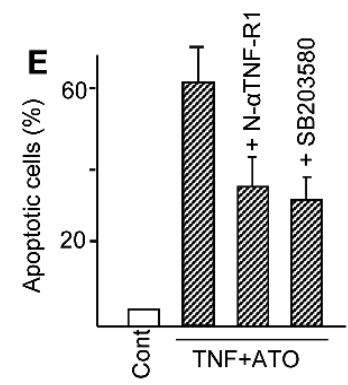

$\mathbf{F}$

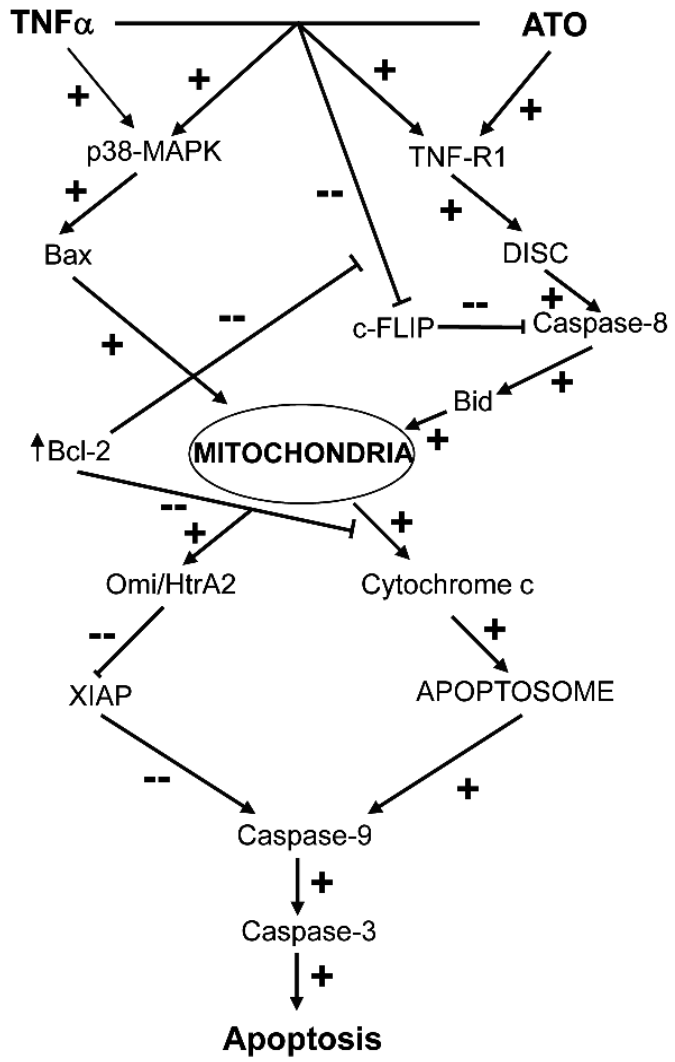

Fig. 2. Cooperative effects between ATO and TNFa. (A) Frequency of apoptosis in U937 cells upon treatment with the indicated concentrations of ATO and TNF $\alpha$, alone and in combination. + Bcl-2, + z-IETD and $+\mathrm{z}-\mathrm{VAD}$ indicate the degree of protection obtained by Bcl-2 transfection or co-treatment with the caspase-8 inhibitor z-IETD-fmk or the pan-caspase inhibitor z-VAD-fmk $(50 \mu \mathrm{M})$. (B, C) Expression of TNF-R1 and c-FLIPL (B) and caspase-8/cleavage activation (C) in cells subjected to the indicated treatments. Observe that Bcl-2 transfection prevents c-FLIPL down-regulation by TNF $\alpha$ plus ATO. (D) Stimulation of $\mathrm{p} 38$-MAPK phosphorylation/activation by $\mathrm{TNF} \alpha$, and its prevention by co-treatment with anti-TNF-R1 neutralizing antibody (N- $\alpha$ TNF-R1). (E) Attenuation of TNF+ATO-provoked apoptosis by co-treatment with N- $\alpha$ TNF-R1 or the p38-MAPK inhibitor SB203580. Except when otherwise indicated, ATO was used at $4 \mu \mathrm{M}$ and $\mathrm{TNF} \alpha$ at $40 \mathrm{ng} / \mathrm{ml}$. Determinations of apoptosis (A, E) were carried out at $24 \mathrm{~h}$ of treatment, and protein analysis (B-D) at $16 \mathrm{~h}$ of treatment. (F) These and other (not shown) results were used to elaborate the present scheme, which describes the interaction between the extrinsic and intrinsic pathways. In summary ATO, alone or with TNF $\alpha$, elicits TNF-R1 expression and c-FLIP inhibition. This facilitates cytokine-provoked activation of the extrinsic (receptor-mediated) pathway, involving caspase- 8 . Complementary TNF $\alpha$, alone or with ATO, causes p38-MAPK activation, which in turn facilitates Bax-mediated activation of the intrinsic (mitochondrial) pathway. Both pathways are mutually interdependent. Thus, caspase- 8 activation engages the mitochondrial pathway via Bid cleavage/activation; and enforced Bcl-2 expression not only prevents mitochondrial activation (e.g., inhibition of cytochrome c release), but also restrains caspase-8 activation by preventing c-FLIP inhibition. Mitochondrial activation involves cytochrome c release, which facilitates apoptosome assembly, and Omi/HtrA2 release, which causes degradation of the inhibitory protein XIAP, both effects leading to the activation of the caspase-9/caspase- 3 pathway. Symbols + and - indicate up-regulation and down-regulation, respectively, of either expression or activity. These results were adapted from Amrán et al. (2007), Ph.D. dissertation, Universidad Complutense de Madrid. 


\section{ARSENIC TRIOXIDE AS A SENSITIZING AGENT}

It is known that apoptosis is executed throughout two main discernible pathways, termed "intrinsic" (mitochondrial) and "extrinsic" (death receptor-mediated) (Fulda and Debatin 2006). In short, the intrinsic pathway, induced by most chemotherapeutic agents, is characterized by the release of cytochrome c and other mitochondria-located proteins to the cytosol, assembly and activation of the apoptosome, degradation of caspase-restraining IAPs ("inhibitor of apoptosis proteins"), and sequential activation of initiator caspase- 9 and effector caspases ( -3 and/or -7). On the other hand the extrinsic pathway, triggered by cytokines of the tumour necrosis factor alpha (TNF $\alpha$ ) family, is initiated by cytokine binding and activation of death receptors (TNF-Rs), followed by DISC ("death-inducing signalling complex") formation and activation of initiator caspase- 8 (or -10) and effector caspases. In some cell types (termed "type I" cells), in which DISC formation is strong enough, intense caspase- 8 activation may suffice to directly elicit effector caspase activation and final apoptosis. By contrast, in cells in which DISC formation is weak (termed "type II" cells), the apoptotic signal requires amplification by mitochondrial engagement, via caspase-8-mediated activation of the Bcl-2-family pro-apoptotic protein Bid.

While the preceding paragraphs were aimed at considering strategies of sensitization to ATO-provoked apoptosis, ATO itself may in turn operate as a sensitizing agent. In fact, in addition to the capacity of ATO to trigger mitochondria-dependent apoptosis by direct binding to mPTP, low drug concentrations may induce death-receptor expression (e.g., Liu et al 2003, Szegezdi et al 2006, Kim et al 2008), thus rendering the cells more responsive to TNF $\alpha$ family cytokines. In a recent study (Amrán et al. 2007), we proposed that co-treatment or pre-treatment with ATO sensitized the otherwise TNF $\alpha$-refractory U937 myeloid leukaemia cells to cytokine-provoked apoptosis, and that sensitization was the result of a complex mechanism involving the interplay of both receptor-mediated and mitochondrial pathways (see some selected results and a schematic representation in Fig. 2). Thus, the combination of ATO plus TNF $\alpha$ efficaciously activated typical events of the mitochondrial executioner pathway, such as release of mitochondrial-located proteins (cytochrome $\mathrm{c}$ and Omi/HtrA2) to the cytosol, Bax translocation to mitochondria, XIAP degradation, and activation of the caspase-9/-3 pathway, while the effects of ATO and TNF $\alpha$ separately were negligible. When upstream regulatory events were investigated, it was observed that ATO (alone or in combination with TNF $\alpha$ ) stimulated receptor type 1 (TNF-R1) expression, decreased the caspase-8 antagonist c-FLIP ("FLICE inhibitory protein") expression, and elicited cleavage/activation of caspase- 8 and Bid. Determinations using appropriate anti-TNF-R1 neutralizing antibody and caspase-8-specific inhibitor demonstrated that activation of the TNF-R1/caspase-8/Bid axis was required for final apoptosis execution. Conversely and importantly, we could observe that enforced Bcl-2 over-expression prevented c-FLIP inhibition and caspase- 8 activation, indicating that proper activation of the extrinsic pathway is under mitochondrial control. Finally (and also importantly), TNF $\alpha$ caused TNF-R1-dependent phosphorylation/activation of $\mathrm{p} 38$-MAPK, and this activation was required for proper activation of the mitochondrial executioner pathway.

While the use of TNF $\alpha$ in anti-tumour therapies is questionable (actually we selected this cytokine only for mechanistic reasons, not for therapeutic considerations), we and others also demonstrated cooperation to induce apoptosis between ATO and the TNF- $\alpha$ family member Apo2L/TRAIL, both in leukaemic (Liu t al. 2003, Szegezdi et al. 2006) and non-leukaemic (Kim et al. 2008) tumour cell models. Apo2L/TRAIL is considered to be a promising anticancer agent because of its capacity to induce apoptosis in cancer cells with lower toxicity towards normal cells (Kimberley et al. 2004), but its efficacy as a single agent is frequently limited. Hence, priming with ATO might represent a valuable strategy to improve the clinical efficacy of this cytokine.

\section{CONCLUSIONS}

In summary, the variety of molecular effects with evident pro-apoptotic consequences makes of ATO a promissory drug for the treatment of multiple neoplastic diseases. Nonetheless, adequate combination with sensitizing agents, often desirable in clinical protocols, may be a sine qua non condition in the case of ATO to conciliate clinical efficacy with low, tolerable side-toxic effects. The well-known susceptibility of ATO toxicity to moderate oxidative stress - either intracellular GSH depletion or increased ROS accumulation - offers an adequate framework for the generation of sensitizing strategies. Among many other examples presented in the literature, we have demonstrated that co-treatment 
with pharmacological protein kinase inhibitors and dietary polyphenols may offer excellent possibilities, and that the sensitizing capacity of these agents is in part mediated by the generation of moderate oxidative stress, at least under in vitro conditions. Conversely, the capacity of ATO to activate apical events of "extrinsic" apoptotic pathway might represent a way of improving the efficacy of cytokines with potential clinical application.

\section{ACKNOWLEDGEMENTS}

This work was supported by the Ministerio de Ciencia e Innovación, Spain, Grant SAF2007-64721 (Plan Nacional de Investigación Científica, Desarrollo e Innovación Tecnológica, Dirección General de Investigación), and Grant CSD2007-00020 (Programa Consolider-Ingenio 2010).

\section{REFERENCES}

Abou-Jawde RM, Reed J, Kelly M, Walker E, Andresen S, Baz R, Karam MA, Hussein M: Efficacy and safety results with the combination therapy of arsenic trioxide, dexamethasone, and ascorbic acid in multiple myeloma patients: a phase 2 trial. Med Oncol 23:263-272, 2006.

Aggarwall BB, Shishodia S: Molecular targets of dietary agents for prevention and therapy of cancer. Biochem Pharmacol 71:1397-1421, 2006.

Altman JK, Yoon P, Katsoulidis E, Kroczynska B, Sassano A, Redig AJ, Glaser H, Jordan A, Tallman MS, Hay N, Platanias LC: Regulatory effects of mammalian target of rapamycin-mediated signals in the generation of arsenic trioxide responses. J Biol Chem 283:1992-2001, 2008.

Amadori S, Fenaux P, Ludwig H, O’Dwyer M, Sanz $\mathrm{M}$ : Use of arsenic trioxide in haematological malignancies: insight into the clinical development of a novel agent. Curr Med Res Opin 21:403-411, 2005.

Amrán D, Sánchez Y, Fernández C, Ramos AM, de Blas E, Bréard D, Calle C, Aller P: Arsenic trioxide sensitizes promonocytic leukaemia cells to TNF $\alpha$-induced apoptosis via p38-MAPK-regulated activation of both receptor-mediated and mitochondrial pathways. Biochim Biophys Acta 1773:1653-1663, 2007.

Anand P, Sundaram C, Jhurani S, Kunnumakkara AB, Agarwall BB: Curcumin and cancer: An "old-age" disease with an "age-old" solution. Cancer Lett 267:133-164, 2008.

Chen D, Chan R, Waxman S, Jing Y: Buthionine sulfoximine enhancement of arsenic trioxide-induced apoptosis in leukaemia and lymphoma cells is mediated via activation of c-Jun NH2-terminal kinase and up-regulation of death receptors. Cancer Res 66:11416-11423, 2006.

Dai J, Weinberg RS, Waxman S, Jing Y: Malignant cells can be sensitized to undergo growth inhibition and apoptosis by arsenic trioxide through modulation of the glutathione redox system. Blood 93:268-277, 1999.

Fernández C, Ramos AM, Sancho $\mathrm{P}$, Amrán D, de Blas E, Aller P: 12-O-tetradecanoylphorbol13-acetate may both potentiate and decrease the generation of apoptosis by the antileukemic agent arsenic trioxide in human promonocytic cells. Regulation by extracellular signal-regulated protein kinases and glutathione. J Biol Chem 279:3877-3884, 2004.

Fulda S, Debatin KM: Extrinsic versus intrinsic apoptosis pathways in anticancer chemotherapy. Oncogene 25:4798-4811, 2006.

Galati G, Sabzevari O, Wilson JX, O'Brien PJ: Prooxidant activity and cellular effects of the phenoxyl radicals of dietary flavonoids and other polyphenolics. Toxicology 177:91-104, 2002.

Garg AK, Buchholz TA, Aggarwall BB: Chemosensitization and radiosensitization of tumours by plant polyphenols. Antioxid Redox Signal 7:1630-1647, 2005.

Kim EH, Yoon MJ, Kim SU, Kwon TK, Sohn S, Choi KS: Arsenic trioxide sensitizes human glioma cells, but not normal astrocytes, to TRAIL-induced apoptosis via CCAAT/enhancerbinding protein homologous protein-dependent DR5 up-regulation. Cancer Res 68:266-275, 2008.

Kim SK, Woodcroft KJ, Khodadadeh SS, Novak RF: Insulin signaling regulates gammaglutamylcysteine ligase catalytic subunit expression in primary cultured rat hepatocytes. $\mathrm{J}$ Pharmacol Exp Ther 311:99-108, 2004.

Kimberley FC, Screaton GR: Following a TRAIL: update on a ligand and its five receptors. Cell Res 14:359-372, 2004.

Lau AT, Wang Y, Chiu JF: Reactive oxygen species: Current knowledge and applications in cancer research and therapeutic. J Cell Biochem 104:657-667, 2008.

Limtrakul P: Curcumin as a chemosensitizer. Adv Exp Med Biol 595:269-270, 2007. 
Lin TH, Lu FJ, Yin YF, Tseng TH: Enhancement of esculetin on arsenic trioxide-provoked apoptosis in human leukaemia U937 cells. Chem Biol Interact 180:61-68, 2009.

Liu Q, Hilsenbeck S, Gazitt Y: Arsenic trioxide-induced apoptosis in myeloma cells: p53-dependent G1 or G2/M cell cycle arrest, activation of caspase- 8 or caspase- 9 , and synergy with APO2/TRAIL. Blood 101:4078-4087, 2003.

Lunghi P, Giuliani N, Mazzera L, Lombardi G, Ricca M, Corradi A, Cantoni AM, Salvatore L, Riccioni R, Constanzo A, Testa U, Levrero $M$ et al.: Targeting the MEK/MAPK signal transduction module potentiates ATO-induced apoptosis in multiple myeloma cells through multiple signalling pathways. Blood 112:2450-2462, 2008.

Maeda H, Hori S, Ohizumi H, Segawa T, KAkehi Y, Ogawa O, Kakizuka A: Effective treatment of advanced solid tumours by the combination of arsenic trioxide and L-buthionine-sulfoximine. Cell Death Differ 11:737-746, 2004.

Miller WH, Jr., Schipper HM, Lee JS, Singer J, Waxman S: Mechanisms of action of arsenic trioxide. Cancer Res 62:3893-3903, 2002.

Mohammad RM, Banerjee S, Li Y, Aboukameel A, Kucuk O, Sarkar FH: Cisplatin-induced antitumour activity is potentiated by the soy isoflavone genistein in BxPC-3 pancreatic tumor xenografts. Cancer 106:1260-1268, 2006.

Nakazato T, Ito K, Ikeda Y, Kizaki M: Grean tea component, catechin, induces apoptosis of human malignant B cells via production of reactive oxygen species. Clin Cancer Res 11:6040-6049, 2005.

Ozben T: Oxidative stress and apoptosis: Impact on cancer therapy. J Pharm Sci 96:2181-2196, 2007.

Pelicano H, Feng L, Zhou Y, Carew JS, Hileman EO, Plunkett W, Keating MJ, Huang P: Inhibition of mitochondrial respiration. A novel strategy to enhance drug-induced apoptosis in human leukemia cells by reactive oxygen species-mediated mechanism. J Biol Chem 278:37832-37839, 2003.

Platanias LC: Biological responses to arsenic compounds. J Biol Chem 284:18583-18587, 2009.

Rahman I, Biswas SK, Kirkham PA: Regulation of inflammation and redox signalling by dietary polyphenols. Biochem Pharmacol 72:1439-1452, 2006.

Ramos AM, Aller P: Quercetin decreases intracellular GSH content and potentiates the apoptotic action of the antileukemic drug arsenic trioxide in human leukemia cell lines. Biochem Pharmacol 75:1912-1923, 2008.
Ramos AM, Fernández C, Amrám D, Sancho P, de Blas E, Aller P: Pharmacologic inhibitors of PI3K/Akt potentiate the apoptotic action of the antileukemic drug arsenic trioxide via glutathione depletion and increased peroxide accumulation in myeloid leukaemia cells. Blood 105:4013-4020, 2005.

Ramos AM, Fernández C, Amrán D, Esteban D, de Blas E, Palacios MA, Aller P: Pharmacologic inhibitors of extracellular signal-regulated kinase (ERKs) and c-Jun NH2-terminal kinase (JNK) decrease glutathione content and sensitize human promonocytic leukaemia cells to arsenic trioxide-induced apoptosis. J Cell Physiol 209:1006-1015, 2006.

Ramos S: Cancer chemoprevention and chemotherapy: dietary polyphenols and signalling pathways. Mol Nutr Food Res 52:507-526, 2008.

Salvi M, Brunati AM, Clari G, Toninello A: Interaction of genistein with the mitochondrial electron transport chain results in opening of the membrane transition pore. Biochim Biophys Acta 1556:187-196, 2002.

Sánchez Y, Amrán D, Fernández C, de Blas E, Aller P: Genistein selectively potentiates arsenic trioxide-induced apoptosis in human leukaemia cells via reactive oxygen species generation and activation of reactive oxygen species-inducible protein kinases (p38-MAPK, AMPK). Int J Cancer 123:1205-1214, 2008.

Sánchez Y, Amrán D, de Blas E, Aller P: Regulation of genistein-induced differentiation in human acute myeloid leukaemia cells (HL60, NB4). Protein kinase modulation and reactive oxygen species generation. Biochem Pharmacol 77:384-396, 2009a.

Sánchez Y, Calle C, de Blas E, Aller P: Modulation of arsenic trioxide-induced apoptosis by genistein and functionally related agents in U937 human leukaemia cells. Regulation by ROS and mitogen-activated protein kinases. Chem Biol Interact 182:37-44, 2009b.

Sarkar FH, Li Y: Using chemopreventive agents to enhance the efficacy of cancer therapy. Cancer Res 66:3347-3350, 2006.

Scott N, Hatlelid KM, MacKenzie NE, Carter DE: Reactions of arsenic(III) and $\operatorname{arsenic}(\mathrm{V})$ species with glutathione. Chem Res Toxicol 6:102-106, 1993.

Sordet O, Rébé C, Leroy I, Bruey JM, Garrido C, Miguet C, Lizard G, Plenchette S, Corcos L, Solary E: Mitochondria-targeting drugs arsenic trioxide and lonidamine bypass the resistance of TPA-differentiated leukemic cells to apoptosis. Blood 97:3931-3940, 2001. 
Sun WM, Huang ZZ, Lu SC: Regulation of gamma-glutamylcyteine synthetase by protein phosphorylation. Biochem J 320:321-328, 1996.

Szegezdi E, Cahill S, Meyer M, O’Dwyer M, Samali A: TRAIL sensitisation by arsenic trioxide is caspase- 8 dependent and involves modulation of death receptor components and Akt. Br J Cancer 94:398-406, 2006.

Tabellini G, Cappellini A, Tazzari PL, Falà F, Billi AM, Manzoli L, Cocco L, Martelli AM: Phosphoinositide 3-kinase/Akt involvement in arsenic trioxide resistance of human leukaemia cells. J Cell Physiol 202:623-634, 2005.

Wang ZY, Chen Z: Acute promylocytic leukaemia: from highly fatal to highly curable. Blood 111:2505-2515, 2008.
Yang CH, Kuo ML, Chen JC, Chen YC: Arsenic trioxide sensitivity is associated with low level of glutathione in cancer cells. $\mathrm{Br} \mathrm{J}$ Cancer 81:796-799, 1999.

Ye J, Li A, Liu Q, Wang X, Zhou J: Inhibition of mitogen-activated protein kinase kinase enhances apoptosis induced by arsenic trioxide in human breast cancer MCF-7 cells. Clin Exp Pharmacol Physiol 32:1042-1048, 2005.

Yi J, Gao F, Shi G, Li H, Wang Z, Shi X, Tang X: The inherent cellular level of reactive oxygen species: one of the mechanisms determining apoptotic susceptibility of leukaemia cells to arsenic trioxide. Apoptosis 7:209-215, 2002. 